Bull. Austral. Math. Soc.

VOL. 41 (1990) [207-213]

\title{
EXPECTED NUMBER OF EXCURSIONS ABOVE CURVED BOUNDARIE. BY A RANDOM WALK
}

\author{
Fima C. Klebaner
}

An asymptotic relation for the expected number of excursions above a boundary $g(n)$ by a random walk $S_{n}, n=1,2, \ldots, N$ is given in terms of an integral involving $g$. An integral test is given to determine whether the total excursion time has finite expectation. If some moment assumptions hold then the expectation of the total excursions is finite if and only if $\int_{1}^{\infty} t^{1 / 2} g^{-1}(t) \exp \left(-g^{2}(t) / 2\right) d t<\infty$.

\section{INTRODUCTION}

Let $X_{1}, X_{2}, \ldots$ be i.i.d. zero mean random variables with finite variance $\sigma^{2}$. Let $S_{N}=\sum_{n=1}^{N} X_{n}$. Let $Y_{N}$ be the number of times $S_{n}$ visits intervals $I_{n}, n=1,2, \ldots, N$. This note studies the asymptotic behaviour of $E Y_{N}$. It is well known that if $I_{n}=I$, a finite interval, then $E Y_{N} \sim C N^{1 / 2}, N \rightarrow \infty$. (see for example Breiman $\left[1\right.$, p.229.]) Here we take $I_{n}$ to be $(g(n), \infty)$, where $g$ is some positive function. It is convinient to write $g(n)=\sigma n^{1 / 2} h(n)$ for some function $h$. We shall assume that $h$ is monotone. In the case when $h(n)=O(1), n \rightarrow \infty$, it is easily seen that $E Y_{N}=\sum_{n=1}^{N} P\left(S_{n}>n^{1 / 2} O(1)\right) \sim C N, N \rightarrow \infty$, as a consequence of the Central Limit Theorem. So we shall assume that $h$ is nondecreasing to $\infty$. In what follows $C$ stands for a positive constant, $F$ and $\psi$ stand for the distribution and the characteristic function of $X_{1}$.

\section{RESULTS}

TheOREM 1. Suppose that $E\left|X_{1}\right|^{5}<\infty$ and that Cramer's condition limsup $|\psi(t)|<1$ holds. Then

$$
E Y_{N} \sim C \int_{1}^{N} h^{-1}(t) \exp \left(-h^{2}(t) / 2\right) d t, N \rightarrow \infty
$$

Received 7 March 1989

Copyright Clearance Centre, Inc. Serial-fee code: 0004-9729/90 \$A2.00+0.00. 
Moreover $C=(2 \pi)^{-1 / 2}$ if the integral diverges or if $F$ is the normal distribution function.

COROLLARY 1. The expected number of visits of $I_{n}$ by $S_{n}, n=1,2, \ldots$ is finite if and only if

$$
\int_{1}^{\infty} h^{-1}(t) \exp \left(-h^{2}(t) / 2\right) d t<\infty
$$

It is interesting to to compare the above criterion (2) with Feller's criterion for the total number of visits, which states that the number of visits of $I_{n}$ by $S_{n}, n=1,2, \ldots$ is finite if and only if

$$
\int_{1}^{\infty} h(t) t^{-1} \exp \left(-h^{2}(t) / 2\right) d t<\infty
$$

See Feller [3, p.211], also Feller [5]. When this is the case it is said that the corresponding function $g$ belongs to the upper class. Note that if $h$ grows to infinity in such a way that the integral in (2) is infinite and the integral in (3) is finite then the total number of excursions above $g$ is almost surely finite but it has infinite mean. The function that provides a boundary between the upper and lower classes is the function from the law of the iterated logarithm, in the sense that for $h(t)$ of the form $(1+\varepsilon)(2 \log \log t)^{1 / 2}$ the integral in (3) converges for $\varepsilon>0$ and diverges for $\varepsilon \leqslant 0$. For functions $h(t)$ of the form $(2 \log t+(1+\varepsilon) \log \log t)^{1 / 2}$ the integral in (2) converges for $\varepsilon>0$ and diverges for $\varepsilon \leqslant 0$. In the spirit of Feller's criterion, (2) may be considered as a criterion to belong to the upper class in the mean.

If $X_{1}$ possesses moments of order less than five then the conclusion of the theorem remains valid for some classes of functions. The method of proof shows how to describe them. Theorem 2 illustrates this. Of course, other generalisations are possible.

Theorem 2. Suppose $E\left|X_{1}\right|^{3}<\infty, \lim \sup |\psi(t)|<1$.

(i) If $h^{-2}(n) \exp \left(h^{2}(n) / 2\right)=O\left(n^{1 / 2}\right), n \rightarrow \infty$, then the integral in (1) diverges and (1) holds.

(ii) If $\sum_{n=1}^{\infty} h^{-3}(n) n^{-1 / 2}<\infty$, then $\lim E Y_{N}=C<\infty$.

COROLlary 2. Suppose (1) holds, $h^{\prime}$ exists and $h(x) h^{\prime}(x) \sim a x^{-1}, x \rightarrow \infty$.

(i) If $0 \leqslant a<1$ then

$$
E Y_{N} \sim(1-a)^{-1} C N h^{-1}(N) \exp \left(-h^{2}(N) / 2\right), N \rightarrow \infty .
$$

(ii) If $a>1$ then the total excursion time has finite expectation. 


\section{EXAmples}

Let $h(x)=\left(\sum_{i=1}^{k} 2 a_{i} \ln _{i} x\right)^{1 / 2}$, where $\ln _{i} x$ is the $i$ th iterate of the function $\ln x$, $x>C$.

1. Let $0 \leqslant a_{1} \leqslant 1 / 2$ and suppose that $X_{1}$ satisfies the conditions of Theorem 2 , or $1 / 2<a_{1}<1$ and $X_{1}$ satisfies the conditions of Theorem 1 . Let $j$ be the first index for which $a_{j} \neq 0$, and take $a_{j}>0$. Then $h(x) h^{\prime}(x) \sim a_{1} / x$ if $a_{1} \neq 0$, and $h(x) h^{\prime}(x)=o(1 / x)$ if $a_{1}=0$. Hence

$$
E Y_{N} \sim C N^{1-a_{1}} \ln _{j}^{-1 / 2} N \prod_{i=j}^{k} \ln _{i-1}^{-a_{i}} N,
$$

where $C=\left(1-a_{1}\right)^{-1}\left(4 a_{j} \pi\right)^{-1 / 2}$.

In particular, the expected number of excursions above $\varepsilon$ in the law of the iterated logarithm by $S_{n}\left(2 \sigma^{2} n \ln _{2} n\right)^{-1 / 2}$ is asymptotically given by $(4 \pi)^{-1 / 2} \varepsilon^{-1} \ln ^{-\epsilon^{2}} N \ln _{2}^{-1 / 2} N$.

2. Let $a_{1}=1$. Then $h(x) h^{\prime}(x) \sim 1 / x$ and this case is not covered by Corollary 2. Take $a_{i}=0$ for $i \geqslant 3$. Evaluation of the integral in (1) gives $E Y_{N} \sim C \ln ^{1 / 2-a_{2}} N$, where $C=(4 \pi)^{-1 / 2}\left(1-a_{2}\right)^{-1}$, provided $a_{2}<1 / 2 ; E Y_{N} \sim C \ln _{2} N$, where $C=$ $(4 \pi)^{-1 / 2}$ if $a_{2}=1 / 2 ;$ and $E Y_{N} \sim C$ if $a_{2}>1 / 2$.

\section{Proofs}

The following lemma is instrumental in the proofs.

Lemma 1. Let $a_{n}=h^{-1}(n) \exp \left(-h^{2}(n) / 2\right), \quad b_{n}=\sum_{k=1}^{3} h^{3 k-1}(n) n^{-k / 2}$ $\exp \left(-h^{2}(n) / 2\right)$. Then

$$
\sum_{n=1}^{\infty} a_{n}<\infty \Rightarrow \sum_{n=1}^{\infty} b_{n}<\infty
$$

and

$$
\sum_{n=1}^{\infty} a_{n}=\infty \Rightarrow \sum_{n=1}^{N} b_{n}=o\left(\sum_{n=1}^{N} a_{n}\right) .
$$

PROOF: Let $\varepsilon>0$ be arbitrary and $u_{n}=h(n) n^{-1 / 6}$.

$$
\sum_{n=1}^{N} b_{n}=\sum_{n=1}^{N} b_{n} I\left(u_{n} \geqslant \varepsilon\right)+\sum_{n=1}^{N} b_{n} I\left(u_{n}<\varepsilon\right) .
$$


To evaluate the first sum in (6) we use the inequality

$$
x^{r} \exp \left(-x^{2} / 2\right) \leqslant(r / e)^{r / 2}, r>0 x \geqslant 0
$$

which is obtained by maximising the function in the left hand side of (7). Choosing $C_{1}=3(14 / e)^{7}$ we obtain for all $x>0$

$$
\sum_{k=1}^{3} x^{3 k-1} \exp \left(-x^{2} / 2\right) \leqslant C_{1} x^{-6}
$$

Using this inequality with $x=h(n)$ we have

$$
b_{n}=\sum_{k=1}^{3} h^{3 k-1}(n) n^{-k / 2} \exp \left(-h^{2}(n) / 2\right)<C_{1} h^{-6}(n) n^{-1 / 2} .
$$

Thus

(9)

$$
\sum_{n=1}^{N} b_{n} I\left(u_{n} \geqslant \varepsilon\right) \leqslant C_{1} \sum_{n=1}^{N} n^{-1 / 2} h^{-6}(n) I\left(u_{n} \geqslant \varepsilon\right)<C_{1} \varepsilon^{-6} \sum_{n=1}^{\infty} n^{-3 / 2}<C_{2} \varepsilon^{-6}<\infty
$$

where $C_{2}=2 C_{1}$. To evaluate the second sum in (6) notice $b_{n}=\left(u_{n}^{3}+u_{n}^{6}+u_{n}^{9}\right) a_{n}$. Hence

$$
\sum_{n=1}^{N} b_{n} I\left(u_{n}<\varepsilon\right)<3 \varepsilon^{3} \sum_{n=1}^{N} a_{n}, \quad \text { if } \varepsilon<1
$$

Let now $\sum_{n=1}^{\infty} a_{n}<\infty$. Then from (6) (9) and (10) it follows that

$$
\sum_{n=1}^{\infty} b_{n}<C_{2} \varepsilon^{-6}+3 \varepsilon^{3} \sum_{n=1}^{\infty} a_{n}<\infty
$$

which is (4). Let $\sum_{n=1}^{\infty} a_{n}=\infty$. For a given $\varepsilon>0$ choose $M$ so large that for all $N \geqslant M$, $C_{2}<\varepsilon^{7} \sum_{n=1}^{N} a_{n}$. Hence from (6), (9) and (10)

$$
\left(\sum_{n=1}^{N} b_{n}\right)\left(\sum_{n=1}^{N} a_{n}\right)^{-1}<\varepsilon+3 \varepsilon^{3}
$$

which is (5). 
Proof of Theorem 1: Denote by $I(A)$ the indicator of set $A$. $\Phi$ and $f$ denote respectively the standard normal distribution and the density functions. We have

$$
Y_{N}=\sum_{n=1}^{N} I\left(S_{n} \epsilon I_{n}\right)=\sum_{n=1}^{N} I\left(S_{n}>\sigma n^{1 / 2} h(n)\right)
$$

and

$$
E Y_{N}=\sum_{n=1}^{N} P\left(S_{n}>\sigma n^{1 / 2} h(n)\right)
$$

Let $F_{n}(x)=P\left(S_{n}<\sigma n^{1 / 2} x\right)$, then proceeding from (11)

$$
E Y_{N}=\sum_{n=1}^{N}\left(1-F_{n}(h(n))\right)=\sum_{n=1}^{N}(1-\Phi(h(n)))+\sum_{n=1}^{N}\left(\Phi(h(n))-F_{n}(h(n))\right)
$$

Using the inequality

$$
a^{-1}\left(1-a^{-2}\right) \exp \left(-a^{2} / 2\right)<\int_{a}^{\infty} \exp \left(-t^{2} / 2\right) d t<a^{-1} \exp \left(-a^{2} / 2\right)
$$

see Feller $[4$, p.175] we obtain

$$
\sum_{n=1}^{\infty}(1-\Phi(h(n)))<\infty \Leftrightarrow \sum_{n=1}^{\infty} h^{-1}(n) \exp \left(-h^{2}(n) / 2\right)<\infty
$$

Moreover, if the series diverges

$$
\begin{gathered}
\sqrt{ }(2 \pi) \sum_{n=1}^{N}(1-\Phi(h(n))) \sim \sum_{n=1}^{N} h^{-1}(n) \exp \left(-h^{2}(n) / 2\right) \\
=\sum_{n=1}^{N} a_{n} \sim \int_{1}^{N} h^{-1}(t) \exp \left(-h^{2}(t)\right) d t, N \rightarrow \infty .
\end{gathered}
$$

To evaluate the second sum in (12) we use the Edgeworth expansion for $F_{n}$.(See Feller $[3$, p.541], Petrov [6, p.159].) Assumptions of the theorem imply

$$
F_{n}(x)=\Phi(x)+f(x) \sum_{k=1}^{3} R_{k}(x) n^{-k / 2}+o\left(n^{-3 / 2}\right)
$$


where $o\left(n^{-3 / 2}\right)$ as $n \rightarrow \infty$ holds uniformly in $x, R_{k}(x)$ is a polynomial of order $3 k-1$, which depends on $F$ only through the first five moments of $F$ and does not depend on $n, k=1,2,3$. Letting $x=h(n)$ in (15) we obtain

$$
\sum_{n=1}^{N}\left(\Phi(h(n))-F_{n}(h(n))\right)=\sum_{n=1}^{N} f(h(n)) \sum_{k=1}^{3} R_{k}(h(n)) n^{-k / 2}+o\left(N^{-1 / 2}\right), N \rightarrow \infty
$$

Since $R_{k}(x)=O\left(x^{3 k-1}\right), x \rightarrow \infty$, we obtain

$$
\sum_{n=1}^{N}\left(\Phi(h(n))-F_{n}(h(n))\right)=O\left(\sum_{n=1}^{N} b_{n}\right)
$$

(1) now follows from (12) by (14), (16) and Lemma 1.

Finally if $F$ is the standard normal distribution function then the second sum in (12) is identically zero and the Theorem follows by (14).

Proof of Theorem 2: Taking the expansion for $F_{n}$ in the form found in Petrov $[6$, p.169]

$$
F_{n}(x)=\Phi(x)+f(x) R_{1}(x) n^{-1 / 2}+Q_{n}(x)
$$

where

$$
\left|Q_{n}(x)\right|<\left(1+|x|^{3}\right)^{-1} o\left(n^{-1 / 2}\right)
$$

with $o\left(n^{-1 / 2}\right), n \rightarrow \infty$ being uniform in $x$. Let $c_{n}=h^{2}(n) n^{-1 / 2} \exp \left(-h^{2}(n) / 2\right)$ and $d_{n}=\left|Q_{n}(h(n))\right|$. Then by (17)

$$
\left|\Phi(h(n))-F_{n}(h(n))\right|<C\left(c_{n}+d_{n}\right)
$$

Since for all $x$ large enough $x^{-2} \exp \left(x^{2} / 2\right)>\exp (x)$, and by the assumption in (i). $h^{-2}(n) \exp \left(h^{2}(n) / 2\right)<C n^{1 / 2}$, we have for all $n$ large enough $h(n)<C \ln n$, which implies that

$$
a_{n}=h^{-1}(n) \exp \left(-h^{2}(n) / 2\right)>C_{1} h^{-3}(n) n^{-1 / 2}>C_{2} n^{-1 / 2} \ln ^{-3} n
$$

and $\sum_{n=1}^{\infty} a_{n}=\infty$. Lemma 1 implies $\sum_{n=1}^{N} c_{n}=o\left(\sum_{n=1}^{N} a_{n}\right)$. From the definition of $d_{n}$ it is seen that $d_{n}=o\left(a_{n}\right), n \rightarrow \infty$. Hence $\sum_{n=1}^{N} d_{n}=o\left(\sum_{n=1}^{N} a_{n}\right)$, and statement (i) of the Theorem now follows by (12) and (14). 
In the second case, due to monotonicity of $h^{-3}(n) n^{-1 / 2}$, convergence of the series $\sum_{n=1}^{\infty} h^{-3}(n) n^{-1 / 2}$ implies $\lim h^{-3}(n) n^{1 / 2}=0$. Hence $h(n)>C n^{1 / 6}$, which implies that $\sum_{n=1}^{\infty} a_{n}<\infty$. By Lemma $1 \sum_{n=1}^{\infty} c_{n}<\infty . \sum_{n=1}^{\infty} d_{n}<\infty$ by the assumption in (ii) and (18). The Theorem now follows from (12), (13) and (19).

Proof of Corollary 2: The proof follows directly from the following Lemma 2.

LEMMA 2. Let $k(x)>0$ be such that $k^{\prime}(x) / k(x) \sim a / x, x \rightarrow \infty$.

(i) If $a>-1$ then $\int_{1}^{\infty} k(t) d t=\infty$ and $\int_{1}^{x} k(t) d t \sim(a+1)^{-1} x k(x), x \rightarrow \infty$.

(ii) If $a<-1$ then $\int_{1}^{\infty} k(t) d t<\infty$.

Notice that if $k(x)=h^{-1}(x) \exp \left(-h^{2}(x) / 2\right)$ then $k^{\prime}(x) / k(x) \sim-h^{\prime}(x) h(x)$ so that Corollary 2 follows. The proof of Lemma 2 can be found in Dieudonne [2, pp.81,82]. It is obtained by integration by parts. $\int_{1}^{x} k(t) d t=x k(x)-k(1)-\int_{1}^{x} t k^{\prime}(t) d t$, and $\int_{1}^{x}\left(k(t)+t k^{\prime}(t)\right) d t=x k(x)-k(1)$. By the assumption $t k^{\prime}(t) \sim a k(t)$, so that $\int_{1}^{x}\left(k(t)+t k^{\prime}(t)\right) d t \sim(a+1) \int_{1}^{x} k(t) d t$ and Lemma 2 follows. The result also holds for $a=0$, see Dieudonne [2] for details.

\section{REFERENCES}

[1] L. Breiman, Probability (Addison-Wesley, Reading, 1968).

[2] J. Dieudonne, Infinitesimal Calculus (Hermann, Paris, 1971).

[3] W. Feller, An Introduction to Probability Theory and Its Applications 1 (John Wiley \& Sons, New York, 1968).

[4] W. Feller, An Introduction to Probability Theory and Its Applications 2 (John Wiley \& Sons, New York, 1971).

[5] W. Feller, 'The general form of the so-called law of the iterated logarithm', Trans. Amer. Math. Soc. 54 (1943), 373-402.

[6] V.V. Petrov, Sums of Independent Random Variables (Springer, Berlin, Heidelberg, New York, 1975).

Department of Statistics

The University of Melbourne

Parkville Vic 3052

Australia 